\title{
Conhecimento dos enfermeiros da Saúde da Família sobre os Transtornos Psíquicos no Período Puerperal ${ }^{1}$
}

The Knowledge of Family Health Nurses' About Psychological Disorders along the Postpartum Time

Conocimiento de los enfermeiros de la Salud de la Familia sobre los transtornos psíquicos en el Período Puerperal

Eveline Ponchet Alves ${ }^{\mathrm{I}}$, Priscilla Maria de Castro Silva ${ }^{\mathrm{II}}$, Elisângela Braga Azevedo ${ }^{\mathrm{III}}$, Maria de Oliveira Ferreira Filha ${ }^{\mathrm{IV}}$

\begin{abstract}
RESUMO
Os transtornos psíquicos puerperais são alterações mentais que podem começar dentro de dias ou semanas após o parto e se manifestam por desequilíbrios de humor psicóticos e não psicóticos. Este trabalho objetivou analisar o conhecimento dos enfermeiros da Saúde da Família sobre os transtornos psíquicos associados ao período puerperal e as intervenções necessárias ao acolhimento dessas demandas. Estudo exploratório-descritivo, realizado com dez enfermeiros de Campina Grande - PB, em 2009. O material empírico foi coletado através de entrevista semiestruturada e tratado através da análise de conteúdo. Os resultados mostraram que os enfermeiros possuem pouco conhecimento sobre os transtornos psíquicos puerperais, desconsiderando a importância da intervenção precoce para minimizar os danos decorrentes dos mesmos para a saúde da mãe e do bebê. Conclui-se que é imprescindível uma atualização para estes profissionais para que possam ter maior conhecimento sobre a temática, intervindo na prevenção desses transtornos e consequentemente fortalecendo o processo de cuidar.
\end{abstract}

Descritores: Transtornos Mentais; Período Pós-Parto; Cuidados de Enfermagem.

\section{ABSTRACT}

The puerperal psychiatric disorders are characterized as mental changes that may start just few days or weeks after childbirth and manifests as psychotic and non-psychotic imbalances of mood. This paper's aim is to analyze nurses' knowledge about psychiatric disorders associated with the postpartum period and interventions necessary to promote health care assistance. This descriptive an exploratory study was carried out with ten nurses from Campina Grande, Paraíba, in 2009. The empirical material was produced from semi-structured interviews and analyzed by content analysis. Results eviudence that nurses have low knowledge about postpartum psychiatric disorders, disregarding the importance of early intervention to minimize damage by the same for the health of mother and baby. We conclude that an update is essential for these professionals so they can have more knowledge on this subject, intervening in the prevention of these disorders and thus strengthening the care process.

Descriptors: Mental Disorders; Postpartum Period; Nursing Care.

\section{RESUMEN}

Los trastornos psíquicos puerperales se caracterizan por cambios mentales que pueden comenzar algunos días o semanas después del parto, y manifiéstense como desequilibrios de humor psicóticos y no psicóticos. En este artículo se objetivó analizar el conocimiento de los enfermeros de Salud de la Familia sobre los trastornos psíquicos asociados al período puerperal. Estudio exploratorio-descriptivo, realizado con diez enfermeros de Campina Grande - PB, en 2009. El material empírico fue obtenido mediante entrevista semiestructurada, y analizado por medio del análisis de contenido. Los resultados mostraron que los enfermeros poseen poco conocimiento sobre los trastornos psíquicos puerperales, desconsiderando la importancia de la intervención precoz para minimizar los daños derivados de los mismos para la salud de la madre y del bebé. Se concluye que es imprescindible una actualización para estos profesionales para que puedan tener mayores conocimientos sobre la temática, interviniendo en la prevención de estos trastornos y consecuentemente fortaleciendo el proceso de cuidar.

Descriptores: Trastornos Mentales; Periodo de Posparto; Atención de Enfermería.

\footnotetext{
${ }^{1}$. Este artigo é um recorte do trabalho de conclusão de curso de enfermagem intitulado: concepções dos enfermeiros da estratégia saúde da família acerca dos transtornos psíquicos no período puerperal, apresentado ao Departamento de Enfermagem da Faculdade de Ciência Médicas de Campina Grande- PB.

${ }^{\text {I }}$ Enfermeira. Campina Grande, PB, Brasil. E-mail: elisaaz@terra.com.br.

II Enfermeira, Discente do Programa de Pós-Graduação em Enfermagem, nível Mestrado, Universidade Federal da Paraíba (UFPB). Professor Auxiliar I, Universidade Federal de Campina Grande. Campina Grande, PB, Brasil. E-mail: priscillamcs@hotmail.com.

${ }^{\text {III }}$ Enfermeira, Mestre em Enfermagem, Discente do Programa de Pós-Graduação em Enfermagem, nível Doutorado, UFPB. Campina Grande, PB, Brasil. E-mail: elisaaz@terra.com.br.

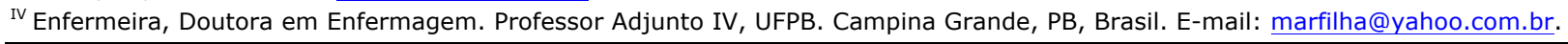




\section{INTRODUÇÃO}

O período pré-natal é considerado um momento de preparação física e psicológica para o parto e para a maternidade. Portanto, torna-se um processo de intenso aprendizado e uma oportunidade para os profissionais da equipe de saúde da família desenvolverem suas práticas consolidando o processo de cuidar, cada vez mais ampliado, visando atender as necessidades das gestantes que procuram os serviços de saúde ${ }^{(1)}$.

A literatura indica que o período gravídico-puerperal é a fase de maior incidência de transtornos psíquicos, necessitando, portanto, de atenção especial para manter ou recuperar o bem estar e prevenir dificuldades futuras para o filho(2). Com isso, os transtornos psíquicos puerperais são definidos como alterações mentais que podem se iniciar dentro de dias ou semanas após o parto e que se manifestam por desequilíbrios de humor psicóticos e não psicóticos ${ }^{(3)}$.

$\mathrm{Na}$ Classificação Internacional de Doenças (CID-10), os transtornos psíquicos puerperais não são considerados distúrbios mentais específicos do puerpério, mas sim, associados a ele, ou seja, o parto atua como um fator desencadeante devido à fragilidade psicológica a qual a mulher está exposta ${ }^{(3)}$. Deste modo, os transtornos psíquicos relacionados ao puerpério são classificados como: Síndrome da Tristeza Pós-Parto ou Disforia Puerperal; Depressão Puerperal ou Pós-Parto e Psicose Puerperal ${ }^{(3-4)}$.

São poucos os estudos existentes na literatura sobre a temática em questão. Um estudo epidemiológico evidenciou que a disforia puerperal ocorre em 50\% a $85 \%$ das mulheres, sendo o quadro leve e transitório e não requer tratamento ${ }^{(4)}$. Já a depressão pós-parto tem uma incidência que varia entre 10 a $15 \%{ }^{(3)}$. A psicose puerperal apresentou uma incidência de 1,1 e 4,0 para cada 1.000 nascimentos ${ }^{(5)} ; 1-2$ para cada $1.000^{(2)}$, sendo esse o transtorno considerado o mais grave.

A etiologia das síndromes psíquicas pós-parto também pode estar associada a fatores hormonais ou orgânicos, predisposição feminina, obstétrico, social e psicológico ${ }^{(3-6)}$. Entretanto, o quadro de depressão pósparto ainda é pouco reconhecido pelos profissionais de saúde e, intervenções simples, no que diz respeito à atenção primária em Saúde Pública, são necessárias e capazes de melhorar a qualidade de vida dessas mães e de seus filhos ${ }^{(7)}$.

Partindo-se destas observações, afirma-se que a gestação, o puerpério e todas as modificações decorrentes do ciclo gravídico-puerperal são eventos na vida da mulher, que necessitam ser avaliados com um olhar mais atento, pois envolvem inúmeras alterações físicas, hormonais e psíquicas que podem refletir diretamente na saúde mental da mesma ${ }^{(5)}$.

Cerca de $1 / 5$ das mulheres no período gestacional e no puerpério apresentam algum tipo de transtorno mental. Sabe-se que uma das maiores preocupações é a insuficiência de diagnósticos precisos ou diagnósticos tardios, focando-se muitas vezes a avaliação da saúde no puerpério, a saúde do bebê( ${ }^{(8)}$. Não se pode esquecer de que as consultas direcionadas às puérperas no pósalta hospitalar ainda não se efetivaram, e muitas mulheres só buscam atendimentos na atenção básica caso ocorra alguma intercorrência ou complicações oriundas do parto.

Nessa perspectiva, as Equipes de Saúde da Família, como estratégia de fortalecimento da atenção básica, devem considerar prioritária a promoção de ações específicas para mulheres no puerpério, uma vez que o ciclo gravido-puerperal pode ser considerado como uma complexa experiência de vida e produtora de vulnerabilidade para a saúde dessas mulheres ${ }^{(9)}$.

Reconhecido como um problema de saúde pública, os transtornos mentais interferem de modo intenso e decisivo na vida social, familiar e profissional das pessoas que sofrem e como qualquer outra doença requer tratamento. Muitas pessoas sofrem em silêncio, seja porque não são assistidas, seja porque os profissionais não diagnosticam corretamente, nem tratam adequadamente.

Todavia, a preocupação em detectar precocemente fatores de risco relacionados com os eventos deste importante ciclo da vida da mulher ainda é pouco considerada pelos enfermeiros que atuam na Saúde da Família. Muitos ainda se sentem inseguros, com pouco conhecimento e experiência para acolher pessoas que desencadeiam transtornos psíquicos puerperais, atuando, algumas vezes, na lógica do encaminhamento, sem envolvimento e acompanhamento das mulheres com este tipo de sofrimento, nas Unidades Básicas de Saúde ${ }^{(7)}$.

Alguns indicadores de sinais e sintomas de sofrimento psíquico durante este período poderiam ser melhor bem pesquisados, pois a depressão puerperal tem sinais indicadores que podem ser investigados desde o período gravídico ${ }^{(10)}$. A psicopatologia puerperal acarreta diversos transtornos ao paciente, familiares e, principalmente, na interação mãe-filho ${ }^{(2)}$. Portanto, avalia-se como sendo de grande relevância que os profissionais de saúde, e os enfermeiros, especificamente, investiguem a história pregressa de 
depressão, presença de sintomas depressivos na gravidez e história familiar de transtornos do humor e de ansiedade, pois estes têm sido identificadas como fatores de risco para transtornos no puerpério(11).

Enfatiza-se que os transtornos psíquicos no período gravídico-puerperal não são comumente diagnosticados e/ou percebidos por parte dos enfermeiros, dificultando a intervenção precoce bem como encaminhamentos adequados para os serviços de referência. Sinais de exaustão e depressão são comumente avaliados como normais pelos profissionais de enfermagem o que possibilita iatrogenias em suas práticas.

Desta forma, esse trabalho objetivou avaliar o conhecimento dos enfermeiros, atuantes nas equipes da Saúde da Família, sobre os transtornos mentais associados ao período puerperal e as intervenções necessárias ao acolhimento dessas demandas, tendo em vista a obtenção de informações para uma melhor qualificação destes profissionais e consequentemente aperfeiçoar a atenção para os aspectos psíquicos do cuidado prestado a mulher no ciclo gravídico puerperal.

\section{CAMINHO METODOLÓGICO}

Estudo exploratório e descritivo, tendo em vista que o tema abordado ainda é pouco pesquisado entre os enfermeiros, carecendo de hipóteses que evidenciem a fragilidade do cuidado na área de saúde mental na atenção básica de saúde, especificamente na área da saúde da mulher com ênfase no ciclo gravídico puerperal.

Foi realizado em Unidades de Saúde da Família USF, do município de Campina Grande, Paraíba, Brasil, com 10 enfermeiros atuantes nesses serviços. Para tanto, foram obedecidos os seguintes critérios de inclusão: estar atuando nas USF, por um período mínimo de um ano, não encontrar-se em situação de férias ou afastamento do trabalho no período de realização das entrevistas e aceitar participar da pesquisa. Como delimitação da amostra utilizou-se o critério de saturação das informações prestadas pelos mesmos.

O material empírico foi produzido por meio de entrevista semiestruturada realizada nas USF, no período de agosto a setembro de 2009. As perguntas foram permeadas pelas seguintes questões norteadoras: Qual seu entendimento sobre os transtornos mentais associados ao período puerperal? Durante sua formação acadêmica ou após esta, foi realizado por você algum treinamento ou capacitação sobre essa temática? Quais as intervenções são realizadas por você visando o acolhimento de puérperas com alterações psíquicas? As entrevistas foram gravadas e posteriormente transcritas, transformadas em textos e analisadas, seguindo a técnica de análise de conteúdo do tipo categorial temática, proposta por Bardin ${ }^{(12)}$. Nesta fase, os textos foram cuidadosamente lidos e agrupados, conforme os critérios de homogeneidade, exaustividade, exclusividade, objetividade e adequação ou pertinência.

Inicialmente, foram criadas três subcategorias temáticas reveladoras dos principais temas contidos nos textos; à medida que o material era analisado emergia a necessidade de agrupar os temas em uma grande categoria temática, aglutinadora das subcategorias: conhecimento escasso, rede de serviços, formação acadêmica e profissional. A grande categoria:

Conhecimento Insuficiente para lidar com os transtornos puerperais, representa o grande achado desta pesquisa e as subcategorias, expressam o conteúdo das falas contidas nos textos analisados. A apresentação das falas consta de recortes dos textos e foram considerados consistentes pois não permitiram dupla interpretação.

O projeto de pesquisa foi encaminhado ao Comitê de Ética do Centro de Ensino Superior e de Desenvolvimento (CESED) tendo sido aprovado em 12 de agosto de 2009, com o número de protocolo: 1990.0.000.405-09.

\section{ANÁLISE E DISCUSSÃo DOS RESULTADOS}

\section{Conhecimento insuficiente para lidar com os transtornos puerperais}

Nesta grande categoria, foram analisadas as informações reveladoras do pouco conhecimento dos enfermeiros sobre os transtornos mentais associados ao ciclo gravídico puerperal, bem como aos encaminhamentos na rede de serviços, e sobre os conhecimentos adquiridos durante a formação acadêmica e profissional. Para a análise do material e apresentação dos resultados, optou-se por colocá-los em três subcategorias: conhecimento escasso, rede de serviços e formação acadêmica.

\section{Conhecimento escasso}

Dentre os 10 entrevistados todos afirmaram que a depressão pós-parto é a patologia da qual eles têm conhecimento nesta fase:

O que eu conheço mais é só a questão da depressão pós- parto [...] (E9). 
Contudo, de acordo com a literatura, existem três tipos de transtornos relacionados a essa fase da vida da mulher, classificados como Tristeza pós-parto, Depressão pós-parto e Psicose pós-parto ${ }^{(3)}$.

Pode-se inferir que a deficiência de conhecimentos específicos sobre as alterações/transtornos psíquicas comumente presentes na vida da mulher no ciclo gravídico puerperal interfere negativamente na qualidade da assistência prestada à puérpera, pois a forma mais leve destes transtornos, que é a tristeza pós-parto, relevante por sua elevada incidência, muitas vezes, passa despercebida pela maioria dos profissionais.

Partindo-se do pressuposto que o enfermeiro é o primeiro profissional a ter o contato com a puérpera, considerou-se fundamental saber se os enfermeiros pesquisados sabiam reconhecer, precocemente, sinais de sofrimento psíquico nas mulheres e a partir disso que intervenções eram comuns entre eles. As respostas de dois enfermeiros a este questionamento indicaram que uma boa anamnese (E6) e abertura de espaços de fala (4) podem facilitar a identificação precoce dos fatores predisponentes.

Um dos estudos encontrados na literatura chama atenção para que alguns indicadores de sinais e sintomas de sofrimento psíquico durante este período devem ser pesquisados, pois a depressão puerperal tem sinais indicadores que podem ser identificados desde o período

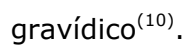

Sabe-se ainda que quando não identificados precocemente e tratados adequadamente, os transtornos do ciclo gravídico puerperal interferem diretamente no relacionamento do binômio mãe-bebê, repercutindo negativamente no desenvolvimento social, afetivo e cognitivo do bebê, e acarretam danos para o desenvolvimento infantil, especialmente no que se refere à posterior ocorrência de problemas emocionais e comportamentais da criança ${ }^{(6-13)}$.

Mesmo com a sutileza com a qual os sintomas psíquicos algumas vezes aparecem, não tornam menos importante suas implicações para a relação mãe-bebê, uma vez que mesmo as formas mais brandas de transtornos psíquicos podem afetar a criança, tendo em vista que ela consegue perceber as mínimas deficiências na contingência do comportamento materno ${ }^{(6)}$.

Avançando-se um pouco mais em busca de identificar que outros conhecimentos os enfermeiros tinham sobre como cuidar da mulher que apresenta alterações psicológicas no ciclo gravídico puerperal, obteve-se as seguintes respostas:
[...] se ela está tendo alteração de comportamento, não só a enfermagem, mas toda a equipe tem que está prestando assistência [...] tem que traçar um plano de assistência, fazer terapia ocupacional, tentar reintegrar a família $[\ldots]$ (E8).

[...] a gente pode oferecer apoio psicológico a essa paciente, encaminhá-la a psicóloga, ao centro de referencia, orientá-la sobre a necessidade desse acompanhamento [...] fazer visitas frequentes para que a gente possa ter uma melhor resposta [...] (E9).

[...] apoio psicológico, conversar com a família, orientar a tomada de medicação correta, tentar amenizar o sofrimento $[\ldots](E 10)$.

As falas acima revelam que os enfermeiros compreendem a importância do processo de trabalho em equipe, da inserção da família no cuidado, e a necessidade de acompanhamento da psicologia de forma mais eficaz durante o período de crise desta puérpera.

Complementando a referida ideia, este estudo revela que o suporte social e emocional dos parentes, em especial o da mãe, nesse momento de grandes mudanças na vida da mulher, como também o apoio social, advindo dos amigos e demais familiares que estão em contato direto com estas mulheres, colaboram para uma progressiva melhora no quadro psíquico ${ }^{(14)}$.

Achados como o infanticídio e o suicídio estão entre as complicações mais graves decorrentes dos transtornos puerperais sem intervenção adequada, porém, a existência de transtornos psiquiátricos não só no puerpério, mas também na gestação, pode levar a outras graves consequências ${ }^{(6)}$. $O$ tratamento médico dos transtornos puerperais deve no mínimo envolver três tipos de atenção: ginecológico, psiquiátrico e psicológico, não podendo deixar de lado os cuidados sociais que comumente estão envolvidos com os casos de depressão pós-parto.

Há necessidade também de se gerar esforços para a realização da educação em saúde como forma de melhorar o impacto dessa ação na saúde física, mental e emocional da gestante durante o pré-natal, quer individualmente ou mesmo coletivamente, sendo importante para isso que a consulta de enfermagem deixe de ser apenas uma consulta mecânica ou apenas um local para esclarecer algumas informações superficiais e que esta seja, de fato, voltada para as demandas e necessidades reais dessa mulher ${ }^{(15)}$.

Sendo assim, é imprescindível que a equipe de saúde esteja preparada para perceber os sinais da doença, para que a partir daí todos os profissionais, de 
maneira interdisciplinar, possam intervir de forma segura e competente, colocando em prática, os princípios do Sistema Único de Saúde em que a assistência prestada deverá ser integral e humanizada.

Recomenda-se que cada caso deve ser avaliado de acordo com sua singularidade, para que seja estabelecida uma melhor estratégia de tratamento para cada situação, o que reforça a necessidade de uma intervenção dos profissionais da saúde direcionada para as demandas maternas que se abrigam por trás da busca do atendimento exclusivo para seus bebês. A visibilidade do sofrimento após o parto poderá prevenir graves problemas pessoais e familiares decorrentes desse tipo de transtorno biopsicoafetivo ${ }^{(16)}$.

\section{Rede de serviços}

Para prestar uma melhor assistência dos casos que não puderam ter resolubilidade na atenção básica, faz-se necessário que os enfermeiros tenham conhecimento acerca da rede de serviços de referência especializada no atendimento aos transtornos psíquicos puerperais. Para tanto, formulou-se a questão: Para quais serviços devem ser encaminhadas as mulheres com alterações mentais?

Considerando que a atenção primária à saúde representa a referência para o primeiro atendimento a puérpera, é de fundamental importância que os profissionais tenham conhecimento dos outros níveis de atenção à saúde e especialidades que possam dar suporte aos casos de difícil resolutividade na Unidade Básica de Saúde, permitindo, com isto, a efetivação da integralidade do cuidado.

Nesta categoria, foram explanados os conhecimentos dos profissionais acerca de qual seria o centro de referência destinado às puérperas com transtornos mentais. Nesse sentido, o grupo de enfermeiros entrevistados relata:

Nós temos o CAPS, mas como nós estamos com o NASF dando esse apoio, então seria primeiramente 0 encaminhamento para a psicóloga(E1).

[...] agora a gente encaminha para psicóloga do NASF e dependendo da necessidade para o serviço de referencia de psiquiatria que seria o CAPS $[\ldots](E 4)$.

[...] o que eu conheço de atendimento são os CAPS(E6). [...] CAPS [...] e agora a gente tem o NASF que é o Núcleo de Apoio à Saúde da Família que dispõe de psicóloga $[\ldots](E 7)$.

Recentemente no município de Campina Grande foi implantado o Núcleo de Apoio à Saúde da Família - NASF
- que, de acordo com a Portaria no 154 de 24 de Janeiro de 2008, tem o objetivo de "ampliar a abrangência e o escopo das ações da atenção básica, assim como sua resolubilidade". É constituído por pelo menos cinco profissionais elencados nas diversas áreas, e distribuídos nas equipes, nas quais se destaca: psicólogo, assistente social, fisioterapeuta, farmacêutico, nutricionista, educador físico, médico pediatra, médico ginecologista, medico psiquiatra, médico acupunturista, médico homeopata, fonoaudiólogo e terapeuta ocupacional.

Em parceria com os profissionais da Equipe de Saúde da Família, o Núcleo de Apoio à Saúde da Família (NASF) - busca atuar de forma que a prática de encaminhamentos para os centros de referências seja reduzida e que casos mais simples sejam resolvidos na própria Unidade Básica de Saúde da Família, evitando, assim, o estrangulamento dos serviços.

O Centro de Atenção Psicossocial (CAPS) representa algo mais que uma mera alternativa ao modelo hospitalar predominante, funcionando de forma a evitar as internações psiquiátricas e diminuir sua reincidência, mas, sobretudo possibilitar o desenvolvimento de laços sociais e interpessoais essenciais para o estabelecimento de novas possibilidades de vida, haja vista que a realização de parcerias entre serviços de saúde é vital para os cuidados em saúde mental no território e para operar os processos de reabilitação psicossocial ${ }^{(17)}$.

Algumas patologias, identificadas no nível primário de saúde, são inviáveis de serem tratadas na atenção básica devido à quantidade restrita de profissionais atuantes nessa área, sendo, desta forma, necessário haver um encaminhamento para os outros níveis de saúde e para que isso aconteça, é indispensável o conhecimento dos profissionais atuantes acerca dos centros de referências existentes.

Uma ação de acolhimento inicial da população para queixas de saúde mental, orientações e informações básicas, assim como, triagem para encaminhamento aos serviços locais disponíveis, também constitui intervenção importante ${ }^{(18)}$.

Nesse contexto, a referência e contra referência representam partes integrantes dos princípios do Sistema Único de Saúde (SUS) que alude ao fato de que a Estratégia Saúde da Família "[...] não é uma peça isolada do sistema de saúde, mas um componente articulado com todos os níveis", possibilitando, dessa forma, melhor conhecimento dos usuários, acompanhamento dos casos, ordenação dos encaminhamentos e a racionalização de recursos tecnológicos do Sistema ${ }^{(15)}$. 
Contudo, nas falas dos entrevistados constatou-se também uma falta de conhecimento sobre o encaminhamento correto, pois um enfermeiro relatou que o encaminhamento deveria ser feito para a maternidade (E2).

Ressalta-se que o enfermeiro deve estar preparado para atender e direcionar, quando necessário, as demandas diversificadas, principalmente quando relacionadas às questões psicológicas que não se evidenciam aparentemente, dificultando ainda mais o diagnóstico preciso e o posterior tratamento. Nesse ínterim, há necessidade do conhecimento do profissional de enfermagem em ter informações sobre os centros de referências disponíveis e existentes na sua região, uma vez que o encaminhamento destas usuárias com demanda de saúde mental para o especialista faz parte do processo de atendimento na perspectiva da integralidade do cuidado.

\section{Formação acadêmica e profissional}

Movidos pela curiosidade em busca de indicadores que revelassem se os enfermeiros tiveram oportunidade de obter tais conhecimentos durante a formação acadêmica, indagou-se sobre conteúdos programáticos que fizeram parte da formação. As respostas indicaram que houve insuficiência de conteúdos para abordar o tema dos transtornos psíquicos vinculados ao ciclo gravídico puerperal.

Nesta perspectiva, pode-se dizer que na maioria dos currículos dos cursos de graduação em enfermagem ainda predomina o modelo biologicista e a fragmentação do conhecimento biopsicossocial.

Durante a vida acadêmica do curso de enfermagem, espera-se que os discentes aprendam conceitos básicos dos transtornos psíquicos em todas as fases do ciclo vital, tornando-os aptos a reconhecer os mais variados quadros clínicos. Neste sentido, quando interrogados se durante a formação acadêmica foi abordado algum assunto relacionado a transtornos psíquicos no ciclo gravídico-puerperal, os discursos dos entrevistados foram unânimes quanto à insuficiência da abordagem dos conceitos básicos sobre os transtornos psíquicos no período da graduação, o que pode ser percebido a seguir:

[...] na Universidade a gente não via muito Saúde Mental não, a não ser a disciplina que a gente pagaria que era Psiquiatria e ali a gente vê tudo, mas não tão aprofundado como exige o programa de Saúde Mental $[\ldots](E 1)$.
[...] se estudei não ficou muito, e olha que não faz muito tempo que eu terminei(E5). Foi visto alguma coisa mais bem superficial, na faculdade $[\ldots](E 8)$.

[...] Saúde Mental especifica para o ciclo gravídico e puerperal não, só no geral mesmo. [...] nem dentro da disciplina de psiquiatria ou de neurologia a gente não viu nada disso (E9). Só no geral, isso é uma coisa bem nova mesmo(E10).

A análise das falas dos profissionais pesquisados evidencia a fragilidade na formação acadêmica. Como consequência da incompletude desse saber teórico, esses profissionais nem sempre estão preparados para identificar ou atender o portador de transtorno psíquico e sua sintomatologia na atenção básica.

Em virtude dessa constatação, tanto neste estudo como em outro $^{(19)}$, constata-se a necessidade de repensar a formação profissional no que tange a alguns aspectos referentes ao ensino das disciplinas de Enfermagem Psiquiátrica e Saúde Mental, a saber: ausência em alguns cursos; redução de carga horária; metodologia; processo ensino-aprendizagem; conteúdo programático que as compõe; articulação entre teoria e prática. Portanto, um olhar acadêmico mais acurado sobre esses aspectos é de fundamental importância na formação destes profissionais.

Permeando o campo da capacitação e/ou treinamento relacionado ao cuidado de enfermagem no tocante aos transtornos do ciclo gravídico-puerperal emergiram falas que apontam para a adoção de uma ação de educação permanente, que atente para as necessidades dos profissionais tendo em vista os problemas da clientela presentes no campo de atuação do saúde da família. Alguns trechos das entrevistas são reveladoras dessas afirmações:

Já participei de capacitação em Saúde Mental no geral para implantação mesmo do Programa de Saúde Mental. Agora a gente precisa ter umas reciclagens [...](E1).

[...] em relação especifica a gestante não, só Saúde Mental no geral (E2).

A Saúde Mental já, agora no caso de doenças puerperais em si, não $[\ldots](E 3)$.

Sobre esse assunto não, a Saúde Mental sim, mas não foi abordado esse assunto [...](E6).

Em Saúde Mental no geral [...](E7).

A partir dessas considerações, foi possível identificar que os entrevistados já realizaram algum tipo de capacitação relacionado à Saúde Mental em geral, não 
especificamente um estudo sobre a fase puerperal, o que confirma a necessidade de acessorias e treinamentos relacionados a essa temática para que a partir daí os profissionais possam prestar uma assistência qualificada junto a esses transtornos psíquicos.

Os trabalhadores da saúde, e os enfermeiros especificamente, referem que não têm tido acesso a informações atualizadas para incorporar os avanços científicos em suas práticas clínicas, nem têm incorporadas as transformações da assistência em saúde mental ${ }^{(7)}$.

A Saúde da Família inclui práticas inovadoras em saúde e requer um novo perfil de profissionais. Tal necessidade conduziu o Ministério da Saúde a instituir os Polos de Capacitação, Formação e Educação Permanente para Saúde da Família, cabe aos Polos, dentre outras atividades, ofertarem treinamentos, objetivando a permanente melhoria da resolutividade da equipe, acompanhar e supervisionar seu trabalho na Unidade de Saúde da Família (USF) ${ }^{(14-15)}$.

Estando a Saúde da Família em constante desenvolvimento, a saúde mental tem sido pouco contemplada nos programas de capacitação, o que muitas vezes dificulta a efetivação da assistência direcionada as demandas das puérperas ${ }^{(20)}$.

Face ao déficit no que tange aos incentivos nessa área e a insuficiente quantidade de materiais e trabalhos voltados a essa problemática, observa-se a dificuldade encontrada por esses profissionais em assistir pacientes com transtornos dessa natureza, uma vez que o desconhecimento e a desinformação é ainda a principal causa de atendimento inadequado e, consequentemente, um sofrimento psíquico da puérpera.

\section{CONSIDERAÇÕES FINAIS}

Neste estudo foi analisado o conhecimento dos enfermeiros atuantes em Unidades de Saúde da Família sobre os transtornos psíquicos que acometem as mulheres no ciclo gravídico puerperal. Particularmente evidenciou-se um problema de saúde pública que acomete uma parcela significativa de mulheres que necessitam serem cuidadas de forma integral.

Utilizando um método qualitativo, este estudo exploratório identificou fragilidades no conhecimento dos enfermeiros que cuidam dessas mulheres, e também revelou uma deficiência na formação acadêmica desses profissionais que ainda não foi preenchida pela educação permanente em saúde.

Os relatos, que convergiram para as informações sobre a formação acadêmica dos entrevistados, foram bastante preocupantes, uma vez que os profissionais saem das instituições de ensino com pouco embasamento teórico, na perspectiva da saúde mental da mulher, dificultando e prejudicando ainda mais a intervenção nesses casos e a assistência prestada nas unidades de saúde.

A partir dos resultados encontrados pode-se afirmar que os enfermeiros ainda não estão devidamente qualificados para prestar uma assistência integral às mulheres com sinais e sintomas de alterações psíquicas puerperais, já que os seus conhecimentos são escassos e restritos a respeito do assunto, o que interfere nas suas condutas.

Os resultados sugerem reflexões, uma vez que é necessária uma coerência entre prática/ensino, para que o cuidado a saúde da mulher se consolide em uma perspectiva ética, humanizada e ampliada, pautada nas necessidades reais destas gestantes e puérperas.

A enfermagem, por estar próxima dessa clientela, em particular, na coordenação de ações junto às equipes de saúde da família, pode e deve atuar na prevenção e controle desses transtornos. Desde o contexto da atenção básica, até os centros de internação para acompanhamento do parto, o enfermeiro é um profissional importante para dar orientações e esclarecimentos, ou até mesmo adotar medidas de intervenção e encaminhamento de mulheres com suspeitas de quadros sugestivos de comprometimento da saúde mental das puérperas.

Nesse contexto, esta pesquisa não esgota as necessidades de estudos mais aprofundados no tocante aos transtornos psíquicos no período puerperal, urge que haja educação permanente nas equipes de saúde da família, para que se possa inicialmente sensibilizar tais profissionais para esta vertente de cuidado e sugere-se neste estudo, que a academia fomente tais ações, já que as pesquisas, e posteriores resultados estão centralizados principalmente nestes sítios.

Sabe-se que a responsabilidade social das Instituições de Ensino Superior é de extrema importância para que as ações de educação em saúde se concretizem. Projetos de pesquisa/extensão desenvolvidos nessa perspectiva podem levar até a comunidade e aos profissionais de saúde, capacitações, informações qualificadas e ações importantes de saúde que subsidiem condutas profissionais para que se obtenham resultados favoráveis após o diagnóstico de tais transtornos. 


\section{REFERÊNCIAS}

1. Rios CTF, Vieira NFC. Ações educativas no pré-natal: reflexão sobre a consulta de enfermagem como um espaço para educação em saúde. Cien Saude Colet [Internet]. 2007 [cited 2011 set 30];12(2):477-86. Available

from:

http://www.scielo.br/pdf/csc/v12n2/a24v12n2.pdf.

2. Falcone VM, Mäder CVN, Nascimento CFL, Santos JMM, Nóbrega FJ. Atuação multiprofissional e a saúde mental de gestantes. Rev Saude Publica [Internet]. 2005 [cited 2011 set 30];39(4):612-8. Available

from:

http://www.scielosp.org/pdf/rsp/v39n4/25534.pdf.

3. Silva ET, Botti NCL. Depressão puerperal - uma revisão de literatura. Rev. Eletr. Enf. [Internet]. 2005 [cited 2011 set 30]; $7(2): 231-8$ Available

from:

http://www.fen.ufg.br/revista/revista7_2/revisao_01.htm.

4. Cantilino A, Zambaldi CF, Sougey EB, Rennó Jr. J. Transtornos psiquiátricos no pós-parto. Rev. psiquiatr. clín. [Internet]. 2010 [cited 2011 set 30];37(6):278-84. Available from: http://www.scielo.br/pdf/rpc/v37n6/a06v37n6.pdf.

5. Camacho RS, Cantinelli FS, Ribeiro CS, Cantilino A, Gonsales BK, Braguittoni É et al. Transtornos psiquiátricos na gestação e no puerpério: classificação, diagnóstico e tratamento. Rev. psiquiatr. clín. [Internet]. 2006 [cited 2011 set 30];33(2):92102. Available

from:

http://www.scielo.br/pdf/rpc/v33n2/a09v33n2.pdf.

6. Schwengber DDS, Piccinini CA. A experiência da maternidade no contexto da depressão materna no final do primeiro ano de vida do bebê. Estud. psicol. (Campinas) [Internet]. 2005 [cited 2011 set 30];22(2):143-56. Available from: http://www.scielo.br/pdf/estpsi/v22n2/v22n2a04.pdf.

7. Kogima EO, Reis AOA. O entendimento dos enfermeiros de uma unidade básica de saúde acerca da depressão puerperal. Nursing (São Paulo). 2009;12(135):381-6.

8. Pereira PK, Lovisi GM. Prevalência da depressão gestacional e fatores associados. Rev. psiquiatr. clín. [Internet]. 2008 [cited 2011 set 30];35(4):144-53. Available from: http://www.scielo.br/pdf/rpc/v35n4/04.pdf.

9. Conill EM, Fausto MCR, Giovanella L. Contribuições da análise comparada para um marco abrangente na avaliação de sistemas orientados pela atenção primária na América Latina. Rev. Bras. Saude Mater. Infant. [Internet]. 2010 [cited 2011 set 30],10 Suppl 1:S14-27. Available from: http://www.scielo.br/pdf/rbsmi/v10s1/02.pdf.

10. Felix GMA, Gomes APR, França PS. Depressão no ciclo gravídico-puerperal. Comun. ciênc. Saúde [Internet]. 2008 [cited 2011 set 30];19(1):51-60. Available from: http://www.fepecs.edu.br/revista/Vol19_1art07.pdf.

11. Andrade LHSG, Viana MC, Silveira CM. Epidemiologia dos transtornos psiquiátricos na mulher. Rev. psiquiatr. clín. [Internet]. 2006 [cited 2011 set 30];33(2):43-54. Available from: http://www.scielo.br/pdf/rpc/v33n2/a03v33n2.pdf.

12. Bardin L. Análise de conteúdo. Lisboa: Edições 70, 2009.

13. Gomes LA, Torquato VS, Feitoza AR, Souza AR, Silva MAM, Pontes RJS. Identificação dos fatores de risco para depressão pós-parto: importância do diagnóstico precoce. Rev. Rene [Internet]. 2010 [cited 2011 set 30],11(esp.):117-23. Available from:

http://www.revistarene.ufc.br/edicaoespecial/a13v11esp_n4.pdf. 14. Cruz EBS, Simões GL, Faisal-Cury A. Rastreamento da depressão pós-parto em mulheres atendidas pelo programa Saúde da Família. Rev Bras Ginecol Obstet [Internet]. 2005 [cited 2011 set 30];27(4):181-8. Available from: http://www.scielo.br/pdf/rbgo/v27n4/a04v27n4.pdf.

15. Rios CTF, Vieira NFC. Ações educativas no pré-natal: reflexão sobre a consulta de enfermagem como um espaço para educação em saúde. Cien Saude Colet [Internet]. 2007 [cited 2011 set 30];12(2):477-86. Available

from: http://www.scielo.br/pdf/csc/v12n2/a24v12n2.pdf. 16. Saraiva ERA, Coutinho MPL. A estrutura das representações sociais de mães puérperas acerca da depressão pós-parto. PsicoUSF (Impr.) [Internet]. 2007 [cited 2011 set 30];12(2):319-26. Available from: http://pepsic.bvsalud.org/pdf/psicousf/v12n2/v12n2a20.pdf.
17. Delfini PSS, Sato MT, Antoneli PP, Guimarães POS. Parceria entre CAPS e PSF: o desafio da construção de um novo saber. Cien Saude Colet [Internet]. 2009 [cited 2011 set 30];14(1):1483-92. Available from: http://www.scielosp.org/pdf/csc/v14s1/a21v14s1.pdf.

18. Bandeira M, Freitas LC, Carvalho Filho JGT. Transtornos mentais comuns em usuários do Programa de Saúde da Família. J Pediatr (Rio J) [Internet]. 2007 [cited 2011 set 30];56(1):41-7. Available from: http://www.scielo.br/pdf/jbpsiq/v56n1/a10v56n1.pdf. 19. Silva TC, kirschbaum DIR. A construção do saber em enfermagem psiquiátrica: Uma abordagem histórico-crítica. SMAD, Rev. Eletrônica Saúde Mental Álcool Drog. (Ed. port.) [Internet]. 2010 [cited 2011 set 30];6(spe):409-38. Available from:

http://www.revistasusp.sibi.usp.br/scielo.php?script=sci_arttext \&pid=S1806-69762010000300004\&Ing=pt.

20. Pereira MAO, Barbieri L, Paula VP, Fraco MSP. Saúde mental no Programa de Saúde da Família: conceitos dos agentes comunitários sobre o transtorno mental. Rev Esc Enferm USP [Internet]. 2007 [cited 2011 set 30];41(4):567-72. Available from: http://www.scielo.br/pdf/reeusp/v41n4/04.pdf.

Artigo recebido em 05.05.2010.

Aprovado para publicação em 23.09.2011.

Artigo publicado em 30.09.2011. 